\title{
Profile of brentuximab vedotin and its potential in the treatment of relapsed or refractory Hodgkin lymphoma
}

This article was published in the following Dove Press journal:

Blood and Lymphatic Cancer:Targets and Therapy

23 May 2012

Number of times this article has been viewed

\section{Sam Mayes'}

Adam Gibb'

Tim Illidge ${ }^{1,2}$

'The Christie Hospital NHS Foundation, Wilmslow Road, ${ }^{2}$ School of Cancer and Enabling Sciences, University of Manchester, Manchester Academic Health Sciences Centre, UK

Correspondence: Tim Illidge School of Cancer and Enabling Sciences, Manchester Academic Health Science Centre, University of Manchester, Manchester, M20 4BX, UK

Tel +44 I6 I446 8III0

Fax +44 I6 I446800I

Email tmi@manchester.ac.uk

\begin{abstract}
Treatment of primary refractory and relapsed classical Hodgkin lymphoma remains challenging, with disappointing results overall and only a minority of patients enjoying a longterm cure. Until recently, antibody therapy has not played any role in the management of this form of Hodgkin lymphoma. The CD30 antigen was first identified in the Hodgkin ReedSternberg malignant cell of Hodgkin lymphoma and emerged as a promising potential target for antibody treatment. The first-generation monoclonal anti-CD30 antibodies proved disappointing with little clinical efficacy. More recently, a novel class of antibody-drug conjugates has emerged. Antibody-drug conjugates offer the potential to target tumor tissue more specifically and deliver potent drug therapies with minimal or less systemic toxicity. Brentuximab vedotin (SGN-35) is one such drug of this class and combines an anti-CD30 monoclonal antibody and the antitubulin agent, monomethyl auristatin E. Initial Phase I studies of brentuximab vedotin showed a promising $52 \%$ overall response rate in relapsed Hodgkin lymphoma, with minimal toxicity. More recently, the pivotal Phase II study in 102 patients demonstrated an overall response rate of $75 \%$ and a complete response rate of $34 \%$. The median duration of response was 6.7 months, but this was extended to 20.5 months in those who achieved a complete response. This review outlines the development of the antibody-drug conjugate, brentuximab vedotin, for relapsed or refractory classical Hodgkin lymphoma.
\end{abstract}

Keywords: Hodgkin lymphoma, brentuximab vedotin, anti-CD30, antibody, conjugate

\section{Need for novel therapeutics in Hodgkin lymphoma}

Hodgkin lymphoma is a lymphoid neoplasm defined by the presence of large binucleated or multinucleated CD30+ malignant Hodgkin Reed-Sternberg (HRS) cells against a background of inflammatory cells. These malignant HRS cells reside in a milieu of reactive inflammatory cells, comprising a small fraction $(0.1 \%-10 \%)$ of the total cell population. ${ }^{1-3}$ Hodgkin lymphoma accounts for approximately $10 \%$ of all lymphomas in western countries and approximately $0.6 \%$ of all cancers diagnosed in the developed world annually. ${ }^{4}$

Whilst advances in combination chemotherapy and reduced field radiotherapy have resulted in high remission rates, leading to Hodgkin lymphoma being considered as one of the most curable of lymphomas, $20 \%-30 \%$ of patients will either relapse after attaining remission, or have primary refractory disease, and fail to enter a complete remission with initial therapy. Such patients are routinely treated with platinumbased salvage regimens, and if chemosensitive, high-dose chemotherapy plus autologous stem cell transplant (ASCT), with up to approximately 50\% of patients having a further relapse. 5,6 Treatment options are extremely limited for patients who 
relapse beyond second-line therapy or have primary refractory disease. The median time to progression for patients who relapse after ASCT was only 3.8 months and associated with a median survival of 26 months in one study, ${ }^{7}$ with $55 \%$ and $32 \%$ overall survival at 3 and 5 years, ${ }^{8}$ respectively, in another. There is clearly an unmet clinical need for novel therapies in Hodgkin lymphoma, both in improving the efficacy of initial therapy, and also in refractory or relapsed disease. Emerging approaches in relapsed and refractory patients have included reduced intensity conditioning and allogeneic bone marrow transplantation in chemosensitive patients. ${ }^{9}$ Novel therapeutics have included molecularly targeted therapies, such as histone deacetylase and proteasome inhibitors, ${ }^{10}$ naked or unlabelled monoclonal antibodies, radiolabeled antibodies or radioimmunotherapy, ${ }^{11}$ and, more recently, antibody-drug conjugates. This review discusses the background, current data, and potential future directions of brentuximab vedotin (SGN035), a novel antibody-drug conjugate of the anti-CD30 monoclonal antibody and the cytotoxic drug, monomethyl-auristatin E (MMAE), in the treatment of Hodgkin lymphoma.

\section{Targeting CD30 in Hodgkin lymphoma}

The CD30 antigen was first identified in the HRS malignant cell of Hodgkin lymphoma. ${ }^{12}$ CD30 is a member of the tumor necrosis factor superfamily ${ }^{13}$ and is expressed in certain malignancies, including Hodgkin lymphoma and anaplastic large cell lymphoma (ALCL), ${ }^{14}$ with normal expression in health confined to a limited number of cell types, including activated lymphocytes. ${ }^{15}$ The function of CD30 is pleiotropic, complex, and context-driven, being implicated in both cell survival and apoptotic signaling. CD30 signaling is mediated via tumor necrosis factor receptor-associated factor (TRAF), dependent and independent NF $\kappa \mathrm{B}$ activation, c-Jun N-terminal kinase, p38 mitogen-activated protein kinase, and extracellular-regulated kinase, ${ }^{16-20}$ and ultimately appears to favor survival of HRS cells.

In view of the high levels of CD30 expression in Hodgkin lymphoma (98.4\% in one series of classical Hodgkin lymphoma), ${ }^{21}$ there is a sound scientific rationale for targeting this antigen, given its high tumor and limited normal tissue expression. An additional theoretical benefit in targeting the HRS cell may be gained by disrupting the complex signaling pathways between the minority component of HRS cells and majority component of supporting stromal and inflammatory cells which comprise the tumor bulk and promote tumor growth and survival. ${ }^{22}$
There have been a number of previous attempts to utilize anti-CD30 monoclonal antibodies to target the CD30 antigen in Hodgkin lymphoma; however, these were largely disappointing, leading to little or no clinical success. The Ber-H2 monoclonal antibody demonstrated efficient targeting of tumor cells but produced no discernible antitumor effect in a small series of patients with advanced Hodgkin lymphoma, ${ }^{23}$ while MDX-060/5F11 (a fully human monoclonal antibody), showed minimal activity, with only $6 \%$ of patients having Hodgkin lymphoma showing a clinical response in a Phase I/II trial. ${ }^{24}$ Parallel development has explored use of the anti-CD30 monoclonal antibody as a vector to deliver a conjugated cytotoxic drug to the target malignant cell, as part of an antibody-drug conjugate. Conjugation of saporin to Ber-H2 (Ber-H2-S06) ${ }^{25}$ and ricin A-chain to $\mathrm{Ki}-4$ (Ki-4.dgA) ${ }^{26}$ represent two antibody-drug conjugates tested in early phase trials. Further development has been limited by the development of human antimouse or antitoxin antibodies, and minimal clinical effect.

The human immunoglobulin gamma-1 and kappa constant regions were genetically fused onto the murine antiCD30 monoclonal antibody AC10, to produce the chimeric monoclonal antibody known as SGN-3027 which forms the carrier for the antibody-drug conjugate, brentuximab vedotin. Preclinical activity was observed with SGN-30 in L540cy Hodgkin lymphoma cells, ${ }^{27}$ and enhanced survival in disseminated Hodgkin lymphoma xenograft models versus nontreated animals, in addition to significant dose-dependent reductions in tumor growth in localized subcutaneous mouse models of disease. ${ }^{27}$ Efficacy was found to be largely dependent on antibody-dependent cellular cytotoxicity (ADCC) as a key antitumor mechanism.

Unfortunately, these promising preclinical results did not translate into meaningful clinical benefits. SGN-30 was administered weekly for 6 weeks at $2-12 \mathrm{mg} / \mathrm{kg}$ in 24 patients with refractory or recurrent CD30-positive malignancy (87.5\% Hodgkin lymphoma) in a Phase I dose escalation study. The treatment was relatively well tolerated, with low human antichimeric antibody formation, but there were only four cases of stable disease in the Hodgkin lymphoma patients (6-16 months' duration), and a single complete response occurred in a patient with ALCL. ${ }^{28}$ Subsequent Phase II studies again failed to demonstrate significant activity in refractory/recurrent Hodgkin lymphoma. ${ }^{29}$ A Phase II trial investigated the combination of SGN-30 with chemotherapy (gemcitabine, vinorelbine, and liposomal doxorubicin), but was closed prematurely when $21.7 \%$ of patients ( 5 of 23 ) who received the combination suffered grade 3-5 pneumonitis, 
with no evidence of a survival benefit. ${ }^{30}$ Thus SGN-30 appeared to have little clinical benefit overall.

\section{SGN-35 development and preclinical results}

In an attempt to enhance its clinical activity, SGN-30 was conjugated to the synthetic dolastatin ${ }^{31}$ analog, MMAE, a tubulin destabilizer, via a valine-citrulline dipeptide linker, ${ }^{32}$ producing cAC10-vc-MMAE (SGN-35), an antibody-drug conjugate with the same avidity as naked SGN-30 for CD30. This antibody-drug conjugate utilizes the immunological specificity of SGN-30 to target the cytotoxic MMAE molecule to CD30-positive HRS cells, and has two important characteristics, namely stability of the conjugate in vivo and the mechanism of drug release at the binding target (see Figure 1).

In vitro studies with CD30-positive cells, including Hodgkin lymphoma cell lines, demonstrated that effective drug delivery occurs, with the antibody-drug conjugate binding to CD30 and subsequent internalization via clathrinmediated endocytosis with trafficking to the lysosomes, where lysis of the peptide linker (valine-citrulline) by enzymes including cathepsin B releases free MMAE inside the cell. ${ }^{33,34}$ Intracellular accumulation can occur at levels much greater than that of the initial treating SGN-35 concentration $(>400 \mathrm{nmol} / \mathrm{L}$ compared with treating concentrations of $6 \mathrm{nmol} / \mathrm{L}$ ), and over time, free MMAE may then permeate through the cell membrane, increasing localized extracellular levels of drug. ${ }^{34}$ Conversely, CD30-negative cell lines treated with SGN-35 did not release detectable levels of drug, suggesting specificity of targeting and reduced normal tissue toxicity. Free extracellular MMAE may then exert action on other cells in the vicinity as a "bystander effect", as observed when CD30-negative Ramos cells were cocultured with CD30-positive cells and SGN-35 at low concentrations $(1 \mu \mathrm{g} / \mathrm{mL})$ resulting in death of both cell populations. ${ }^{34}$ As a comparison, a nonbinding control antibody-drug conjugate (IgG-vc-MMAE) resulted in death of neither population, underlining the importance of immunologically specific binding, internalization, and intracellular processing for both drug activation and the bystander effect.

The stability of an antibody-drug conjugate is important to enable efficient targeting of the cytotoxic drug to malignant cells with minimal systemic release and exposure to nontarget tissues, thereby reducing toxicity and allowing greater drug loading for a localized effect. Additionally, theoretical concerns exist that an unstable antibody-drug conjugate could result in masking of target sites by the naked monoclonal antibody if deconjugation occurs, thus reducing the availability for remaining stable antibody-drug conjugate, and hence diminishing efficacy. The valine-citrulline

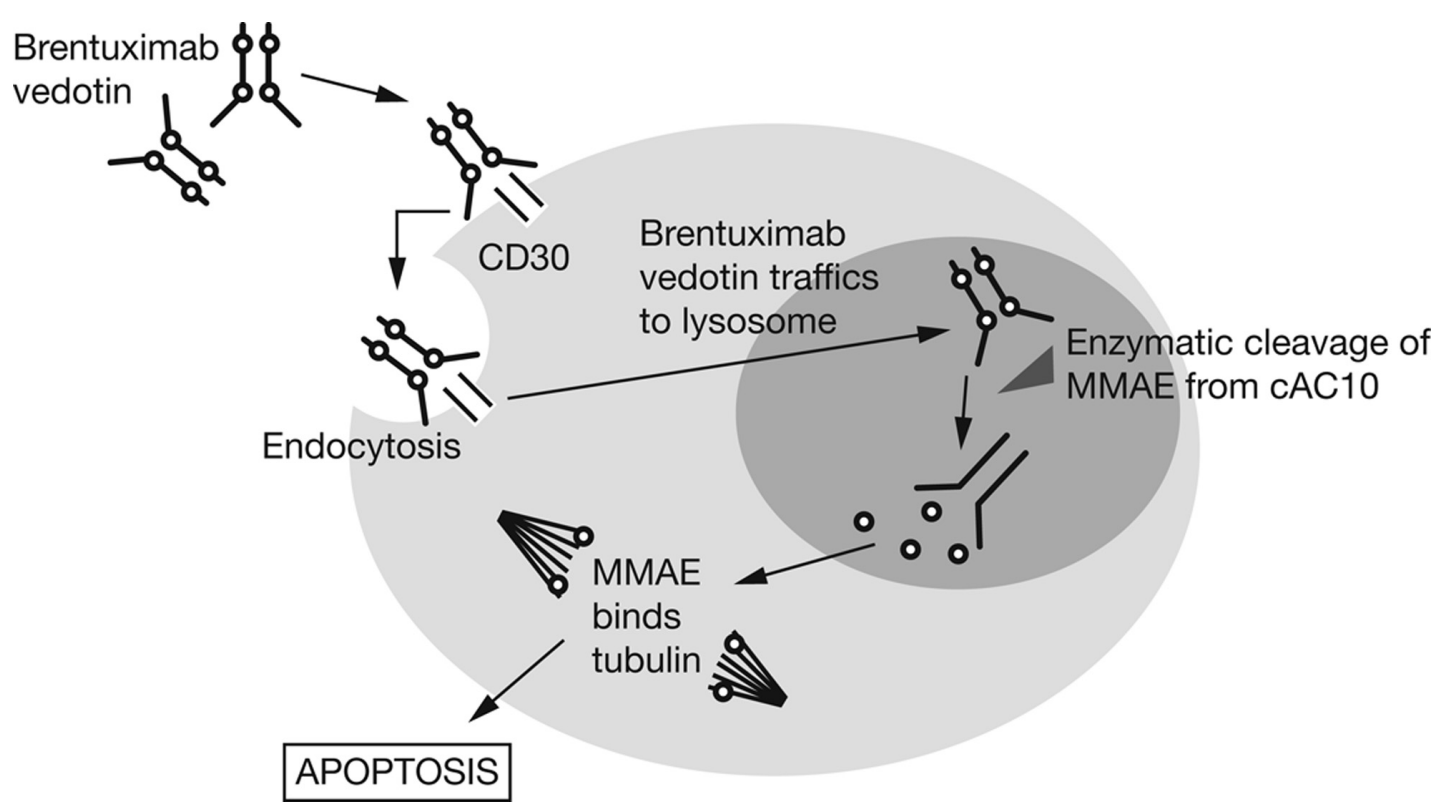

Figure I Brentuximab vedotin is an antibody-drug conjugate (ADC) combining momomethyl auristatin E (MMAE) - a potent antimicrotubule agent - and an anti-CD30 $\mathrm{mAb}$ via a protease cleavable linker. The figure illustrates the steps in ADC action beginning with ADC binding CD30 on target cell, and ultimately intracellular release and cytotoxic action. Free MMAE released into the tumour micro-environment can exert a 'bystander effect' on other cells including supporting stromal cells, likely contributing to the anti-tumour effect.

Reused with permission. Publisher and Licensee Libertas Academica Ltd. Permission to reproduce is subject to license 20I205I5/UK/RTRF.45 
dipeptide linker technology has been engineered to provide such stability in SGN-35, compared with previous hydrazone linkers used for example in the first licensed antibody-drug conjugate, gemtuzumab-ozogamicin $(50 \%$ conjugated forms). ${ }^{35}$ When incubated in human plasma at $37^{\circ} \mathrm{C}$ for 10 days and analyzed for the presence of free drug, less than $2 \%$ was evident, nor were drug or peptide linker degradation/ metabolite products, providing a projected half-life of at least 250 days. ${ }^{32}$ Furthermore, monoclonal antibody-val-citlinked conjugates with differing specificity have been demonstrated elsewhere to have a half-life of 230 days in human plasma compared with 2.6 days for hydrazone-linked conjugates. ${ }^{36}$ Yet more evidence of this stability was seen when anti-CD30 hydrazone-linked conjugates incubated with CD30-negative cells resulted in time-dependent cell death as free drug was liberated, but no cytotoxicity was evident, even after 96 hours, with cAC10-vc-MMAE. Similarly, other experiments have noted a lack of generation of free MMAE in CD30-negative culture. ${ }^{34}$ Taken together, these data indicate that the combination of $\mathrm{cAC} 10$, stable peptide linking technology, and MMAE imparts a high degree of immunological specificity to the antibody-drug conjugate with minimal systemic release, hence minimal toxicity, therefore fulfilling important criteria for an antibody-drug conjugate.

Preclinical studies of SGN-35 in Hodgkin lymphoma (and ALCL, where effects tend to be more pronounced due to increased homogeneity of CD30 expression as compared with Hodgkin lymphoma) have shown pronounced activity. In vitro experiments demonstrated enhanced efficacy at sub CD30-saturating concentrations $(10 \mu \mathrm{g} / \mathrm{mL})$ compared with SGN-30 in L540 Hodgkin lymphoma cells ( IC $_{50} 9.88$ ng/ $\mathrm{mL}$ ) and Karpass 299 ALCL cells $\left(\mathrm{IC}_{50} 2.5 \mathrm{ng} / \mathrm{mL}\right),{ }^{32}$ although an $\mathrm{IC}_{50}$ of $302 \mathrm{ng} / \mathrm{mL}$ in HDLM2 Hodgkin lymphoma cells suggests that rate of internalization, trafficking, or cleavage of free drug may vary within CD30-positive cells and influence efficacy. None of the cell lines treated demonstrated greater than $20 \%$ cytotoxicity with uncross-linked SGN-30 at concentrations up to $1 \mu \mathrm{g} / \mathrm{mL}$. Similar observations have been seen elsewhere for L450cy Hodgkin lymphoma cells $\left(\mathrm{IC}_{50}\right.$ of $\left.63 \mathrm{ng} / \mathrm{mL}\right)$ and KM-H2 HD cells $\left(\mathrm{IC}_{50}\right.$ $90 \mathrm{ng} / \mathrm{mL}),{ }^{36}$ where the mechanism underlying an antitumor effect appears to be G2/M-phase arrest followed by apoptosis.

In vivo experiments have also yielded striking results. The maximum tolerated dose in SCID mice was determined to be $30 \mathrm{mg} / \mathrm{kg}$, and when L540cy xenografts were treated with $1 \mathrm{mg} / \mathrm{kg}(\mathrm{q} 4 \mathrm{~d} \times 4)$ there were partial regressions seen in all animals, ie, at 1/30th of the maximum tolerated dose $\mathrm{e}^{32}$ (at this dose in ALCL xenografts, all animals experienced complete, durable tumor regressions). Only slight tumor growth delay was seen with $1 \mathrm{mg} / \mathrm{kg}$ of unmodified cAC10, and little to no activity with $10 \times$ the molar equivalent of free MMAE. It is also worth noting that in ALCL xenograft models, the combination of free MMAE (equivalent to $10 \times$ that delivered by $1 \mathrm{mg} / \mathrm{kg}$ of antibody-drug conjugate) and $1 \mathrm{mg} / \mathrm{kg}$ of cAC10 produced no detectable antitumor activity, providing a powerful illustration of the importance of stable MMAE conjugation and specific targeting. Elsewhere, complete tumor regressions in L540cy xenografts have been achieved with doses as low as $2 \mathrm{mg} / \mathrm{kg}$ on the same dosing schedule, ${ }^{37}$ and good results have been achieved in treating disseminated Hodgkin lymphoma xenografts.

SGN-35 in combination with ABVD chemotherapy was demonstrated to improve outcomes without increased toxicity in L450cy Hodgkin lymphoma xenograft models. ${ }^{38}$ Treatment with either ABVD or SGN-35 produced significant tumor growth delay, in comparison to control, with $0 / 8$ animals treated with Adriamycin, bleomycin, vinblastine, dacarbazine (ABVD), and 4/9 with SGN-35 (given at $1 \mathrm{mg} / \mathrm{kg} \mathrm{q} 4 \mathrm{~d} \times 3$ ) having durable responses. The combination of SGN-35 and ABVD resulted in 9/9 durable tumor regressions and statistically significant tumor growth delay in comparison with each single treatment arm $(P<0.0101$ versus SGN-35 and $P<0.0001$ versus ABVD). Furthermore, when treatment was instigated at higher tumor burdens, combination therapy significantly increased tumor growth delay and provided durable remissions in 5/10 animals. Similar improvements in activity were observed when SGN-35 was combined with gemcitabine, but this was not seen with other cytotoxic agents such as vinorelbine, suggesting enhanced combination treatment outcomes are chemotherapy-specific. An interesting observation was a lack of increased morbidity or loss of body weight in the ABVD-gemcitabine combination groups, suggesting that increased efficacy can be achieved with little added toxicity. However, this must be interpreted with caution, because SGN-35 does not crossreact with rodent CD30, and in light of the increased pulmonary toxicity observed in the clinic with SGN-30 in combination with gemcitabine-containing chemotherapy. The mechanism underlying the increased efficacy of combination SGN-35 chemotherapy is not clear, but the authors postulate a mechanism whereby SGN-35 eliminates the minority Reed-Stenberg cell population, and the chemotherapy interferes with the supporting cellular infiltrate, in addition to the differing mechanisms of action of the 
cytotoxic drugs (eg, SGN-35 inducing G2/M-phase growth arrest and gemcitabine causing S-phase arrest).

Further refinement of the antibody-drug conjugate occurred when the effect of drug loading per unit monoclonal antibody was explored, with two, four, and eight molecules of MMAE per antibody (E2, E4, and E8, respectively) analyzed. ${ }^{39}$ The differential loading had no effect on ability to bind CD30, and the E4 drug provided a two-fold increase in therapeutic ratio by virtue of increased maximum tolerated dose yet equivalent efficacy to E8. The E4 drug is now known as SGN-35, or brentuximab vedotin.

Taken together, these data provide strong preclinical evidence for the use of SGN-35 in Hodgkin lymphoma as an antibody-drug conjugate that is well tolerated, extremely stable, active at doses which are fractions of the maximum tolerated dose. SGN-35 may elicit cell kill via a number of mechanisms, including direct signal induction, effector cell recruitment, and ADCC, in addition to targeted delivery of a potent cytotoxic drug which has powerful bystander effects that in theory could disrupt the stromal infiltrate in Hodgkin lymphoma, thus rendering the Reed-Sternberg cell less viable.

\section{Clinical experience}

Well over 1000 patients have now been treated worldwide with brentuximab vedotin to date, within a variety of clinical trials and, more recently, a larger number have been treated as part of compassionate use and a named patient program. Three pivotal studies have so far been reported, and the results of those including patients with Hodgkin lymphoma are outlined below (SGN35-001 and 003). Several more trials are currently recruiting subjects in Hodgkin lymphoma (SGN35-005, 009).

The initial Phase I study of brentuximab vedotin investigated a 3-week dosing schedule in 45 patients with relapsed or refractory CD30-positive malignancies, including Hodgkin lymphoma $(\mathrm{n}=42)$, ALCL $(\mathrm{n}=2)$, and angioimmunoblastic T cell lymphoma $(n=1) .{ }^{40}$ The median age of patients recruited into this study was just 36 (range 20-87) years, with Eastern Cooperative Oncology Group performance status of 0 or $1(93 \%)$. Patients were heavily pretreated with a median of three prior chemotherapy regimens (range 1-7), with 73\% having received prior ASCT. The primary study endpoint was determination of the maximum tolerated dose, whilst evaluation of the overall response rate and pharmacokinetics were secondary endpoints. Brentuximab vedotin was administered as an outpatient intravenous infusion at doses ranging from 0.1 to $3.6 \mathrm{mg} / \mathrm{kg}$ every 21 days. Tumor response assessments were performed between the second and third cycles of therapy. Patients with stable disease or a better response after two cycles of therapy were able to continue treatment. Brentuximab vedotin was generally well tolerated at doses up to $1.8 \mathrm{mg} / \mathrm{kg}$, which was defined as the maximum tolerated dose. Dose-limiting toxicities of fatal febrile neutropenia and grade 3 hyperglycemia were observed in the $3.6 \mathrm{mg} / \mathrm{kg}$ and $2.7 \mathrm{mg} / \mathrm{kg}$ cohorts, respectively.

Other common adverse events reported were generally grade 1 or 2 in severity, and included fatigue (16 patients, $36 \%$ ), peripheral neuropathy or related limb pain (16 patients, $36 \%$ ) pyrexia (15 patients, 33\%), and diarrhea, nausea, and neutropenia (10 patients, $22 \%$ each). At the $1.2 \mathrm{mg} / \mathrm{kg}$ dose (one level below the maximum tolerated dose) there were no grade 3 or 4 adverse events. Grade 3 neutropenia, back pain, and limb pain each occurred in one patient out of $12(25 \%)$ at the $1.8 \mathrm{mg} / \mathrm{kg}$ dose level. At doses higher than $1.8 \mathrm{mg} / \mathrm{kg}$, grade 3 neutropenia and pyrexia were seen in two of $12(17 \%)$ patients who received the $2.7 \mathrm{mg} / \mathrm{kg}$ dose. A single patient who received the $3.6 \mathrm{mg} / \mathrm{kg}$ dose developed febrile neutropenia and died of infectious complications. Peripheral neuropathy and limb pain was reported in 16 patients (36\%), 13 of whom were treated at the $1.8 \mathrm{mg} / \mathrm{kg}$ or $2.7 \mathrm{mg} / \mathrm{kg}$ dose, and typically presented with grade 1 or 2 paresthesia in the hands or feet. Three patients discontinued treatment because of peripheral neuropathy. Resolution of peripheral neuropathy was noted in 10 of 16 patients $(63 \%)$ at the last safety assessment for the study.

The objective response rate across all patients and dose cohorts was $38 \%$ (17 of 45 patients), with $24.4 \%$ (11 of 45 patients) achieving a complete response. The 42 Hodgkin lymphoma patients demonstrated an objective response rate of $35.7 \%(15 / 42)$ and complete response rate of $21.4 \%(9 / 42)$. Six of $12(50 \%)$ of patients who received the maximum tolerated dose of $1.8 \mathrm{mg} / \mathrm{kg}$ achieved an overall response, with a median response duration of 9.7 months (range 0.6 to $>19.5$ months). Tumor regression was noted in 36 of $42(86 \%)$ evaluable patients, and 13 of 16 patients $(81 \%)$ with disease-related symptoms at baseline (pruritus, night sweats) had resolution of their symptoms on therapy, irrespective of their antitumor response status.

A second Phase I trial to evaluate the safety and efficacy of a more frequent dosing schedule was performed in patients with CD30-positive malignancies. ${ }^{41} \mathrm{~A}$ total of 37 patients, 31 with Hodgkin lymphoma, five with systemic ALCL, and one with peripheral $\mathrm{T}$ cell lymphoma were enrolled. The median age was 35 (range 13-82) years, and patients had received a median of three prior chemotherapy regimens (range 1-8). Sixty-two percent of patients had had a previous 
ASCT. Patients received brentuximab vedotin weekly for 3 of 4 weeks, with doses ranging from 0.4 to $1.4 \mathrm{mg} / \mathrm{kg}$. The most common treatment-related adverse events included peripheral neuropathy, nausea, fatigue, neutropenia, diarrhea, and dizziness. At $1.4 \mathrm{mg} / \mathrm{kg}$, the maximum tolerated dose was exceeded. The overall response rate evaluated across all dose levels was $46 \%$ (16 of 35 patients), with $29 \%$ (10 of 35 ) patients achieving a complete response, and a median duration of response of 16 weeks.

Given the high response rates in both these Phase I trials, a Phase II trial was conducted in patients with relapsed or refractory Hodgkin lymphoma. The pivotal SGN35-003 Phase II study enrolled 102 subjects with relapsed or refractory Hodgkin lymphoma following the failure of ASCT. The results of this study were initially reported at the American Society of Hematology annual meeting in 2010 and were recently updated. ${ }^{42}$ This study treated 102 patients with relapsed or refractory Hodgkin lymphoma at a dose of $1.8 \mathrm{mg} / \mathrm{kg}$ of brentuximab vedotin every 3 weeks. The demographic features of these patients included a median age of 31 years, Eastern Cooperative Oncology performance status of 0 or 1 , a median of four prior therapies, and all patients had undergone previous ASCT. Seventy-one percent were refractory to frontline therapy and $42 \%$ to their most recent treatment. All were treated with brentuximab vedotin $1.8 \mathrm{mg} / \mathrm{kg}$ until either progression, failure of tolerance, or 1 year (16 cycles) of therapy was complete. The objective response rate was $75 \%$, and the complete response rate was $34 \%$ (see Figure 2). The median duration of response was
6.7 months, but this extended to 20.5 months in those who achieved a complete response. Treatment-emergent adverse events included peripheral sensory neuropathy, fatigue, nausea, and neutropenia (see Table 1), but the majority of these were mild to moderate. Grade $3 / 4$ toxicity was primarily hematological, with $\geq 5 \%$ of subjects experiencing at least one episode of neutropenia, peripheral neuropathy, thrombocytopenia, or anemia. Adverse events led to discontinuation of brentuximab vedotin in approximately $20 \%$ of subjects.

These compelling clinical results led to the accelerated approval of brentuximab vedotin (Adcetris ${ }^{\mathrm{TM}}$ ) by the US Food and Drug Administration in 2011 for the treatment of relapsed or refractory Hodgkin lymphoma in patients with progressive disease after ASCT, or after two chemotherapy regimens in patients ineligible for transplantation. Brentuximab vedotin was also approved for patients with ALCL who have failed at least one prior treatment. Brentuximab vedotin is the first new therapy approved for the treatment of Hodgkin lymphoma in over 30 years.

While the Phase II response data are impressive, the clinical community requires durable remissions, or better still, using the drug as a "bridge" to enable patients to undergo potentially curative transplant procedures. These latter two overlapping goals need to be put into the context of costeffectiveness, given the potentially serious financial implications of prolonged administration of brentuximab vedotin for health care providers. In the context of inducing prolonged remissions, recent emerging data using brentuximab vedotin

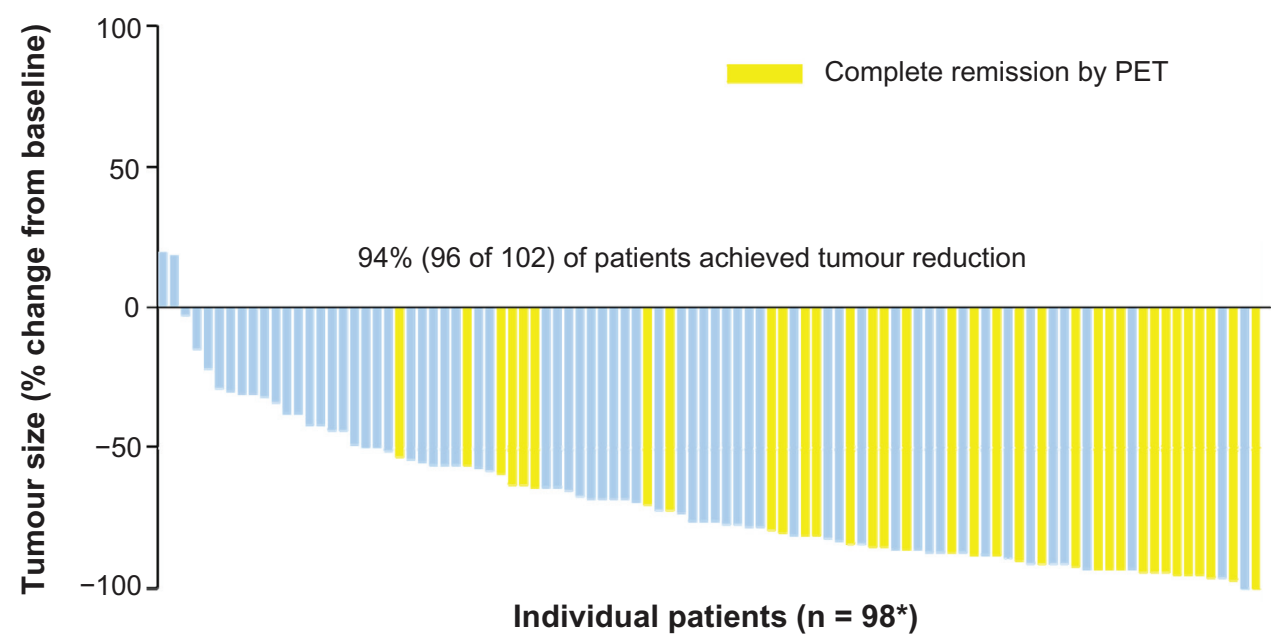

Figure 2 Waterfall plot from Phase II study ${ }^{42}$ demonstrating percentage change in tumor size from baseline following brentuximab vedotin. Ninety-four percent of patients achieved tumor reduction. The objective response rate was $75 \%$ and the complete response rate was $34 \%$. Four patients were not included in the analysis due to lack of measurable lesions or no post-baseline scans.

Reprinted with permission. @ 2012 American Society of Clinical Oncology. All rights reserved.Younes, A et al: Results of a Pivotal Phase II Study of Brentuximab Vedotin for Patients With Relapsed or Refractory Hodgkin's Lymphoma. J Clin Oncol. 2012 Mar 26. [Epub ahead of print].

Abbreviation: PET, positron emission tomography. 
Table I Percentage occurrence of adverse events in Phase II trial of brentuximab vedotin ${ }^{42}$

\begin{tabular}{ll}
\hline Adverse event (all grades) & $\begin{array}{l}\text { Frequency of } \\
\text { occurrence }\end{array}$ \\
\hline Peripheral sensory neuropathy & $47 \%$ \\
Fatigue & $46 \%$ \\
Nausea & $42 \%$ \\
Upper respiratory tract infection & $37 \%$ \\
Diarrhea & $36 \%$ \\
Pyrexia & $29 \%$ \\
Neutropenia & $22 \%$ \\
Vomiting & $22 \%$ \\
Cough & $21 \%$ \\
Common Grade 3 or 4 adverse events & Grade 3, Grade 4 \\
Neutropenia & $14 \%, 6 \%$ \\
Peripheral sensory neuropathy & $9 \%, 0 \%$ \\
Thrombocytopenia & $6 \%, 2 \%$ \\
Anemia & $5 \%, 1 \%$ \\
\hline
\end{tabular}

to induce remission prior to reduced intensity conditioning allogeneic hematopoietic cell transplantation (RIC-allo HCT) has been investigated in the setting of relapsed/refractory Hodgkin lymphoma. ${ }^{43}$ RIC-allo HCT is potentially curative, but is limited by lack of disease control prior to transplantation in this patient population. The impact of brentuximab vedotin on RIC-allo HCT was retrospectively analyzed in 16 patients treated at two centers (12 and four patients, respectively). Conditioning regimens included fludarabinemelphalan (12 patients), fludarabine-Cytoxan/2Gy total body irradiation (three patients), and 2 Gy total body irradiation (one patient), with various graft versus host disease prophylaxis regimens. Progression-free survival was $90 \%$ at 1 year and $100 \%$ at median 7.2 months follow up in twelve and four patient centers, respectively, with overall survival of $100 \%$, and non relapse mortality of $0 \%$. The single relapse was observed in a patient who had progressive disease at the time of engraftment, with 276 days between last brentuximab vedotin administration and RIC-allo HCT. Acute and chronic graft versus host disease rates were $25 \%$ and $63 \%$, respectively. There were no delays in engraftment, and these data demonstrate that SGN-35 can provide prolonged disease control in the setting of RIC-allo HCT without increased complications or graft versus host disease.

\section{Summary of clinical trial experience}

The published data so far clearly indicate that brentuximab vedotin is an extremely active agent as monotherapy, even in patients whose disease has become refractory to combination chemotherapy including high-dose chemotherapy/ASCT. The majority of patients have experienced clinical benefit, and overall response rates have been high, which have translated into durable remissions with extended progression-free survival, which is particularly valuable for patients whose disease is incurable by current established treatments. These durable single-agent response rates are particularly impressive when considering the relative ease of administration by a 3-weekly outpatient short intravenous infusion and the relative lack of significant toxicity and associated high quality of life (with the exception of peripheral neuropathy), especially when compared with standard multiagent chemotherapy regimens used in the "salvage" setting and other novel approaches, including histone deacetylase inhibitors.

\section{Ongoing studies}

Brentuximab vedotin is presently being investigated as a maintenance strategy in patients who are at high risk of relapse following ASCT in a trial known as AETHERA (ADC Empowered Trial for Hodgkin to Evaluate pRogression after ASCT). AETHERA (SGN35-005, NCT01100502) is a randomized, blinded, placebo-controlled Phase III study comparing brentuximab vedotin versus best supportive care immediately following ASCT for relapsed Hodgkin lymphoma. These subjects remain at high risk (about 50\%) of relapse despite ASCT,, 5 and no current standard of care exists to reduce this. Eligible patients receive brentuximab vedotin $1.8 \mathrm{mg} / \mathrm{kg}$ or placebo every 3 weeks for 1 year. Subjects who progress have the option of unblinding, and if on the placebo arm, may then receive brentuximab vedotin in a parallel study (SGN35-010, NCT01196208).

A Phase I trial of brentuximab vedotin in combination with ABVD in untreated Hodgkin lymphoma patients (SGN35-009, NCT01060904) has been completed and was reported at the recent 2011 American Society of Hematology meeting. ${ }^{44}$ Patients were randomized between brentuximab vedotin plus either ABVD or AVD, with a cohort-based design to find the maximum tolerated dose for brentuximab vedotin in combination therapy (not yet reached). With up to $30 \%$ of advanced stage Hodgkin lymphoma patients ultimately experiencing treatment failure with ABVD, improved therapies are urgently needed for this patient group.

\section{Future directions}

Such highly active therapies with manageable toxicity and convenient patient administration are unusual in oncology, and there can be little doubt that brentuximab vedotin will play an important future role in the management of patients 
with Hodgkin lymphoma, as well as ALCL (not covered in this review). However, future challenges await this new highly active and novel therapy. Further information is required regarding managing the long-term toxicities (primarily neurotoxicities and perhaps other as yet potential unknown problems) in patients with few other treatment options. Most critically, further insights will be required on how best to integrate this new therapy into the management of Hodgkin lymphoma at earlier time points, and which combination chemotherapy drugs to combine it with, using established or modified multidrug combinations, such as ABVD and BEACOPP (bleomycin, etoposide, doxorubicin, cyclophosphamide, vincristine, procarbazine, and prednisone).

The high response rates reported in the Phase II pivotal trial were in the context of heavily pretreated patients who had previously been treated with many lines of chemotherapy, including high-dose chemotherapy and ASCT. Using the drug in first-line or earlier lines of therapy is therefore likely to result in higher activity. However, more important is that these properties may allow brentuximab vedotin to be integrated into multiagent chemotherapy regimens, either in addition to or instead of other drugs, creating a new more efficacious and less toxic treatment platform. This possibility of course will require rigorous clinical trial development and it will potentially take many years to achieve this goal, because prolonged efficacy in Phase III trials, including long-term safety analysis, will be required. However, the possibility remains of reducing treatment failure in all lines of therapy for Hodgkin lymphoma (and other CD30-positive malignancies), and achieving maximal tumor cytoreduction. Many future trials have been proposed which may accrue patient benefit, including the use of brentuximab vedotin in the salvage setting with chemotherapy prior to ASCT in relapse. The current standard salvage chemotherapy regimens have a low complete response rate, typically around $25 \%-30 \%$, despite a higher overall response rate. Improving the complete response rate may allow more relapsed/refractory patients to proceed to successful transplantation. The role of brentuximab vedotin in combination with immunomodulatory strategies or in combination with other novel targeted agents, such as NFкB inhibitors, are important directions for future investigations, as well as novel combinations with immunomodulatory agents, such as lenalidomide, in refractory disease. As we gain better scientific understanding of the immunological tumor microenvironment and mechanisms of tumor resistance, we will become increasingly able to design treatment combinations which reflect the new insights gained from the laboratory that can be translated to the clinic. Finally, improvements in outcome are urgently required for the second peak of incidence in Hodgkin lymphoma that occurs in the elderly. Brentuximab vedotin as frontline monotherapy for the elderly or in combination with chemotherapy that has nonoverlapping toxicity has the potential to increase the therapeutic ratio. Such approaches may derive significant clinical benefit and lead to improved outcomes for this important group of patients who currently tolerate and respond poorly to multiagent chemotherapy regimens.

\section{Disclosure}

TI has provided consultancy for Seattle Genetics, Millenium, and Takeda Oncology, and has received honoraria in this capacity.

\section{References}

1. Aldinucci D, Gloghini A, Pinto A, De Filippi R, Carbone A. The classical Hodgkin's lymphoma microenvironment and its role in promoting tumour growth and immune escape. J Pathol. 2010;221(3): 248-263.

2. Steidl C, Connors JM, Gascoyne RD. Molecular pathogenesis of Hodgkin's lymphoma: increasing evidence of the importance of the microenvironment. J Clin Oncol. 2011;29(14):1812-1826.

3. Hsi ED. Biologic features of Hodgkin lymphoma and the development of biologic prognostic factors in Hodgkin lymphoma: tumor and microenvironment. Leuk Lymphoma. 2008;49(9):1668-1680.

4. Ries L, Kosary C, Hankey B, et al, editors. SEER Cancer Statistics Review, 1973-1994, National Cancer Institute. Bethesda, MD: National Institutes of Health: 1997.

5. Sureda A, Constans M, Iriondo A, et al. Prognostic factors affecting long-term outcome after stem cell transplantation in Hodgkin's lymphoma autografted after a first relapse. Ann Oncol. 2005;16(4):625-633.

6. Majhail NS, Weisdorf DJ, Defor TE, et al. Long-term results of autologous stem cell transplantation for primary refractory or relapsed Hodgkin's lymphoma. Biol Blood Marrow Transplant. 2006;12(10):1065-1072.

7. Kewalramani T, Nimer SD, Zelenetz AD, et al. Progressive disease following autologous transplantation in patients with chemosensitive relapsed or primary refractory Hodgkin's disease or aggressive nonHodgkin's lymphoma. Bone Marrow Transplant. 2003;32(7): 673-679.

8. Martinez C, Canals C, Alessandrino E, et al. Relapse of Hodgkin's lymphoma (HL) after autologous stem cell transplantation (ASCT): Prognostic factors in 462 patients registered in the database of the EBMT. ASCO Meeting Abstracts. 2010;28(Suppl 15):8060.

9. Thomson KJ, Peggs KS, Smith P, et al. Superiority of reduced-intensity allogeneic transplantation over conventional treatment for relapse of Hodgkin's lymphoma following autologous stem cell transplantation. Bone Marrow Transplant. 2008;41(9):765-770.

10. Jona A, Younes A. Novel treatment strategies for patients with relapsed classical Hodgkin lymphoma. Blood Rev. 2010;24(6):233-238.

11. Schnell R, Dietlein M, Staak JO, et al. Treatment of refractory Hodgkin's lymphoma patients with an iodine-131-labeled murine anti-CD30 monoclonal antibody. J Clin Oncol. 2005;23(21):4669-4678.

12. Schwab U, Stein H, Gerdes J, et al. Production of a monoclonal antibody specific for Hodgkin and Sternberg-Reed cells of Hodgkin's disease and a subset of normal lymphoid cells. Nature. 1982;299(5878): 65-67.

13. Clodi K, Younes A. Reed-Sternberg cells and the TNF family of receptors/ligands. Leuk Lymphoma. 1997;27(3-4):195-205. 
14. Stein H, Mason DY, Gerdes J, et al. The expression of the Hodgkin's disease associated antigen $\mathrm{Ki}-1$ in reactive and neoplastic lymphoid tissue: evidence that Reed-Sternberg cells and histiocytic malignancies are derived from activated lymphoid cells. Blood. 1985;66(4):848-858.

15. Podack ER, Strbo N, Sotosec V, Muta H. CD30 - governor of memory T cells? Ann NY Acad Sci. 2002;975:101-113.

16. Harlin H, Podack E, Boothby M, Alegre ML. TCR-independent CD30 signaling selectively induces IL-13 production via a TNF receptor-associated factor/p38 mitogen-activated protein kinasedependent mechanism. J Immunol. 2002;169(5):2451-2459.

17. Duckett CS, Gedrich RW, Gilfillan MC, Thompson CB. Induction of nuclear factor kappaB by the CD30 receptor is mediated by TRAF1 and TRAF2. Mol Cell Biol. 1997;17(3):1535-1542.

18. Zheng B, Fiumara P, Li YV, et al. MEK/ERK pathway is aberrantly active in Hodgkin disease: a signaling pathway shared by CD30, CD40, and RANK that regulates cell proliferation and survival. Blood. 2003;102(3):1019-1027.

19. Gruss HJ, Ulrich D, Dower SK, Herrmann F, Brach MA. Activation of Hodgkin cells via the CD30 receptor induces autocrine secretion of interleukin-6 engaging the NF-kappabeta transcription factor. Blood. 1996;87(6):2443-2449.

20. Mir SS, Richter BW, Duckett CS. Differential effects of CD30 activation in anaplastic large cell lymphoma and Hodgkin disease cells. Blood. 2000;96(13):4307-4312.

21. von Wasielewski R, Mengel M, Fischer R, et al. Classical Hodgkin's disease. Clinical impact of the immunophenotype. Am J Pathol. 1997;151(4):1123-1130.

22. Maggio E, van den Berg A, Diepstra A, Kluiver J, Visser L, Poppema S. Chemokines, cytokines and their receptors in Hodgkin's lymphoma cell lines and tissues. Ann Oncol. 2002;13(Suppl 1):52-56.

23. Falini B, Flenghi L, Fedeli L, et al. In vivo targeting of Hodgkin and Reed-Sternberg cells of Hodgkin's disease with monoclonal antibody Ber-H2 (CD30): immunohistological evidence. Br J Haematol. 1992;82(1):38-45.

24. Ansell SM, Horwitz SM, Engert A, et al. Phase I/II study of an anti-CD30 monoclonal antibody (MDX-060) in Hodgkin's lymphoma and anaplastic large-cell lymphoma. J Clin Oncol. 2007;25(19): 2764-2769.

25. Falini B, Bolognesi A, Flenghi L, et al. Response of refractory Hodgkin's disease to monoclonal anti-CD30 immunotoxin. Lancet. 1992;339(8803):1195-1196.

26. Schnell R, Staak O, Borchmann P, et al. A Phase I study with an anti$\mathrm{CD} 30$ ricin A-chain immunotoxin (Ki-4.dgA) in patients with refractory CD30+ Hodgkin's and non-Hodgkin's lymphoma. Clin Cancer Res. 2002;8(6):1779-1786.

27. Wahl AF, Klussman K, Thompson JD, et al. The anti-CD30 monoclonal antibody SGN-30 promotes growth arrest and DNA fragmentation in vitro and affects antitumor activity in models of Hodgkin's disease. Cancer Res. 2002;62(13):3736-3742.

28. Bartlett NL, Younes A, Carabasi MH, et al. A phase 1 multidose study of SGN-30 immunotherapy in patients with refractory or recurrent CD30+ hematologic malignancies. Blood. 2008;111(4): 1848-1854.

29. Forero-Torres A, Leonard JP, Younes A, et al. A Phase II study of SGN30 (anti-CD30 mAb) in Hodgkin lymphoma or systemic anaplastic large cell lymphoma. Br J Haematol. 2009;146(2):171-179.

Blood and Lymphatic Cancer: Targets and Therapy

\section{Publish your work in this journal}

Blood and Lymphatic Cancer: Targets and Therapy is an international, peer-reviewed, open access journal focusing on blood and lymphatic cancer research, identification of therapeutic targets and the optimal use of preventative and integrated treatment interventions to achieve improved outcomes, enhanced survival and quality of life for the cancer
30. Blum KA, Jung SH, Johnson JL, et al. Serious pulmonary toxicity in patients with Hodgkin's lymphoma with SGN-30, gemcitabine, vinorelbine, and liposomal doxorubicin is associated with an FcgammaRIIIa-158 V/F polymorphism. Ann Oncol. 2010;21(11): 2246-2254.

31. Pettit GR. [The dolastatins]. Fortschr Chem Org Naturst. 1997;70:1-79. German.

32. Francisco JA, Cerveny CG, Meyer DL, et al. cAC10-vcMMAE, an anti-CD30-monomethyl auristatin E conjugate with potent and selective antitumor activity. Blood. 2003;102(4):1458-1465.

33. Sutherland MS, Sanderson RJ, Gordon KA, et al. Lysosomal trafficking and cysteine protease metabolism confer target-specific cytotoxicity by peptide-linked anti-CD30-auristatin conjugates. J Biol Chem. 2006;281(15):10540-10547.

34. Okeley NM, Miyamoto JB, Zhang X, et al. Intracellular activation of SGN-35, a potent anti-CD30 antibody-drug conjugate. Clin Cancer Res. 2010;16(3):888-897.

35. Bross PF, Beitz J, Chen G, et al. Approval summary: gemtuzumab ozogamicin in relapsed acute myeloid leukemia. Clin Cancer Res. 2001;7(6):1490-1496.

36. Doronina SO, Toki BE, Torgov MY, et al. Development of potent monoclonal antibody auristatin conjugates for cancer therapy. Nat Biotechnol. 2003;21(7):778-784.

37. Hamblett KJ, Barton J, Cerveny CG, et al. SGN-35, an anti-CD30 antibody-drug conjugate, exhibits potent antitumor activity for the treatment of CD30+ malignancies. ASH Annual Meeting Abstracts. 2005;106(11):610

38. Oflazoglu E, Kissler KM, Sievers EL, Grewal IS, Gerber HP. Combination of the anti-CD30-auristatin-E antibody-drug conjugate (SGN-35) with chemotherapy improves antitumour activity in Hodgkin lymphoma. Br J Haematol. 2008;142(1):69-73.

39. Hamblett KJ, Senter PD, Chace DF, et al. Effects of drug loading on the antitumor activity of a monoclonal antibody drug conjugate. Clin Cancer Res. 2004;10(20):7063-7070.

40. Younes A, Bartlett NL, Leonard JP, et al. Brentuximab vedotin (SGN-35) for relapsed CD30-positive lymphomas. $N$ Engl J Med. 2010;363(19):1812-1821.

41. Fanale M, Bartlett NL, Forero-Torres A, et al. The antibody-drug conjugate brentuximab vedotin (SGN-35) induced multiple objective responses in patients with relapsed or refractory CD30-positive lymphomas in a Phase 1 weekly dosing study. ASH Annual Meeting Abstracts. 2009;114(22):2731.

42. Chen RW, Gopal AK, Smith SE, et al. Results from a pivotal phase II study of brentuximab vedotin (SGN-35) in patients with relapsed or refractory Hodgkin lymphoma (HL). ASCO Meeting Abstracts. 2011;29(Suppl 15):8031.

43. Chen RW, Forman SJ, Palmer J, et al. Brentuximab vedotin (SGN-35) enables successful reduced intensity allogeneic hematopoietic cell transplantation in relapsed/refractory Hodgkin lymphoma. ASH Annual Meeting Abstracts. 2011;118(21):664.

44. Younes A, Connors JM, Park SI, Hunder NNH, Ansell SM. Frontline therapy with brentuximab vedotin combined with ABVD or AVD in patients with newly diagnosed advanced stage Hodgkin lymphoma. ASH Annual Meeting Abstracts. 2011;118(21):955.

45. Furtado M, Rule S. Emerging Pharmacotherapy for Relapsed or Refractory Hodgkin's Lymphoma: Focus on Brentuximab Vedotin. Clin Med Insights Oncol. 2012;6:31-39

\section{Dovepress}

patient. The manuscript management system is completely online and includes a very quick and fair peer-review system. Visit http://www. dovepress.com/testimonials.php to read real quotes from published authors. 